\title{
Photochemical benzylic radical arylation promoted by supported Pd nanostructures
}

Received 00th January 20xx,

\author{
Teresa A. Gawargy, Paolo Costa, Anabel E. Lanterna* and Juan C. Scaiano*
}

Accepted 00th January 20xx

DOI: $10.1039 / \times 0 \times x 00000 x$

We report a novel way to promote photochemical benzylic radical arylations using $\mathbf{P d}$ nanostructures. Traditional benzylic radical reaction pathways are challenged by the presence of metal centres that provoke unprecedent regioselectivity towards more synthetically relevant $\mathrm{C}\left(\mathrm{sp}^{3}\right)-\mathrm{C}\left(\mathrm{sp}^{2}\right)$ couplings. This new $\mathrm{C}-\mathrm{H}$ activation pathway is rationalised by means of a pseudo-persistent radical effect facilitated by metal centres. We show the mechanistic and computational aspects of the heterogeneous photocatalysis processes that are the root of this drastic change in reactivity.

\section{Introduction}

Free radical reactions have long been established as a direct method for carbon-carbon bond formation ${ }^{1}$. In particular, the generation of carbon-centred radicals from $\mathrm{C}-\mathrm{H}$ bond activation is a promising alternative to promote processes that maximize atom and step economy2, 3 . Extensive research in the field has developed novel methods for radical reactions using homogeneous radical initiators (e.g., triethylborane ${ }^{4}$, tert-butyl hydroperoxide ${ }^{5}$, di-tert-butylperoxide ${ }^{6}$ ) and, more recently, examples employing heterogenous semiconductors (e.g., $\mathrm{TiO}_{2}{ }^{7}$, $\left.{ }^{8}\right)$ have proven advantages such as no generation of debris and easy separation. Due to their particular stability comparing to other carbon-centred radicals, benzyl radical intermediates are important in the synthesis of natural products ${ }^{9}$. Free benzyl and related radicals tend to have simple reaction pathways dominated by self-reaction, addition to unsaturated systems ${ }^{5,6}$ and oxidative processes ${ }^{5}$ leading to alcohols, aldehydes and esters ${ }^{10}$. The formation of $\mathrm{C}\left(\mathrm{sp}^{3}\right)-\mathrm{C}\left(\mathrm{sp}^{2}\right)$ bonds, and the novel structures that can derive from these reactions is not a typical pathway for free benzylic radicals. Finding ways to selectively favour non-traditional radical couplings would greatly increase

\footnotetext{
a. Department of Chemistry and Biomolecular Sciences and Centre for Advanced Materials Research (CAMaR), University of Ottawa, 10 Marie Curie, Ottawa, Ontario K1N 6N5, Canada.

Electronic Supplementary Information (ESI) available: Experimental details (catalyst information, product studies, photolysis of dibenzyl ketone) and computational analysis. See DOI: 10.1039/x0xx00000x
}

the use of radical-radical couplings in organic synthesis. Here we show that metal nanoparticles (i.e., PdNP) can change completely the regioselectivity of benzyl radical reactions, from conventional head-to-head combination to efficient head-toring coupling processes (Scheme 1). We propose that benzyl radicals can associate with the PdNP surface drastically changing their reactivity. The interaction with the Pd surface can diminish the "radical" nature of the molecule, an overall effect that can be compared to the persistent radical effect (PRE). ${ }^{11}$

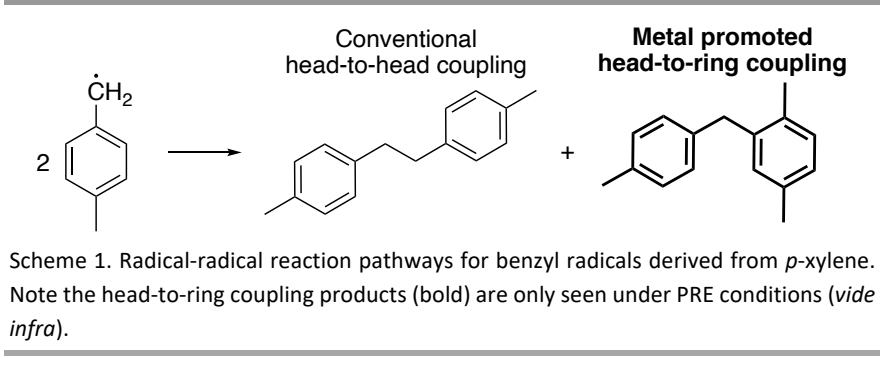

\section{Results and discussion}

Whereas there are different ways to generate free radicals, we have recently reported the use of photoexcited $\mathrm{TiO}_{2}$ as a clean free radical initiator (no initiator debris formed). ${ }^{7}$ Here, we used this method to generate benzyl radicals by oxidation of $p$-xylene through the photogenerated $\mathrm{TiO}_{2}$-hole, an exceptional electrophilic species. This is, the $\mathrm{VB}$ edge of $\mathrm{TiO}_{2}$ ( $2.5 \mathrm{~V}$ vs NHE) appears to have sufficient energy for oxidizing solvents such as toluene ( $2.5 \mathrm{~V}$ vs NHE), $p$-xylene (2.25 V vs NHE). ${ }^{12}$ Scheme 2 shows the proposed mechanism for the formation of benzyl radicals upon UVA excitation of $\mathrm{Pd@TiO}$, which was explained as a dystonic proton-coupled electron-transfer (PCET) process. ${ }^{7}$ In the absence of metal nanoparticles (i.e., Pd), the reaction furnishes only the head-to-head coupling products (Table 1), resembling the behaviour when benzylic radicals are produced in solution by conventional methods - such as in the photolysis of dibenzyl ketone (DBK, Scheme 3) - where the head-to-head 
recombination yields 1,2-diphenylethane. That is, under UVB light irradiation, dibenzyl ketone is decomposed into benzyl radicals and carbon monoxide, generating only the head-head product (See ESI). However, if benzylic radicals are formed in the presence of metal nanoparticles (i.e., $\mathrm{Pd} @ \mathrm{TiO}_{2}$ ), ring recombination leading to 1,4-dimethyl-2-(4methylbenzyl)benzene becomes a major pathway. As expected, the addition of metal nanoparticles increases not only the selectivity towards head-to-ring coupling products $(O P)$ but also the overall rate of the reaction, going from $0.36 \mu \mathrm{mol} / \mathrm{h}$ on bare $\mathrm{TiO}_{2}$ to 0.5 amd $0.6 \mu \mathrm{mol} / \mathrm{h}$ for PdNP@TiO 2 and AuNP@TiO respectively. Table 1 (and Table S2-S3) summarises the reaction of methylbenzenes under UVA irradiation and inert atmosphere using different $\mathrm{TiO}_{2}$ (P25)-based photocatalysts. Details of the reaction conditions as well as catalyst characterization are presented in the accompanying ESI. Chart 1 shows the mechanistic abbreviations used for the different species considered in this work. When bare $\mathrm{TiO}_{2}$ is used as catalyst the reaction leads to a majority of the head-to-head product (BB) whereas only traces of the head-to-ring coupling products (OP and $\boldsymbol{p P}$ ) are seen. Conversely, when the catalyst is replaced by $\mathrm{Pd} @ \mathrm{TiO}_{2}$, the addition of PdNP induces the formation of the oP and $\boldsymbol{p P}$ coupling products, which suggests that the interaction between the metal NP and benzylic radicals promotes head-toring coupling in benzylic systems (Tables 1, S2-S3). Additionally, we determined that the presence of oxidised $\mathrm{Pd}$ species in the material is not detrimental of the formation of head-to-ring products, i.e., we subject the $\mathrm{Pd@TiO}$ to a known reducing treatment ${ }^{13}$ (See ESI), and determined the oxidation state of the Pd species by XPS analysis (Figure S3). As depicted in table S2, selectivity towards head-to-ring coupling remains similar to that found with the fresh (more oxidised) catalyst.

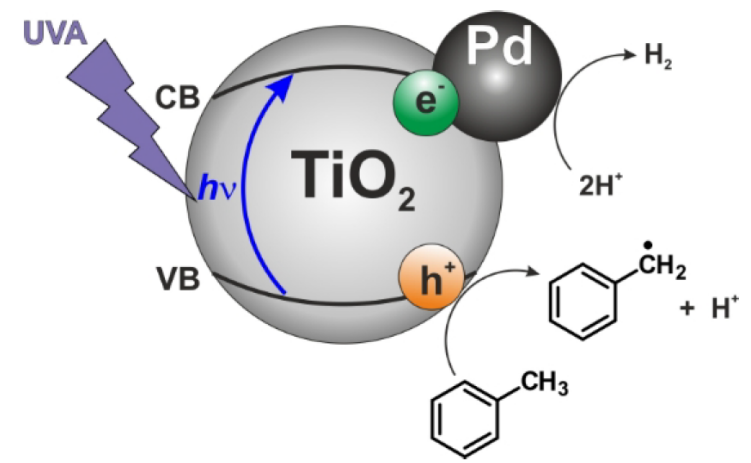

Scheme 2. UVA photoinitiated generation of benzyl radicals from the toluene used as solvent. The PCET process is accompanied by hydrogen gas evolution. A similar mechanism applies for $p$-xylene. VB: valence band. CB: conduction band.

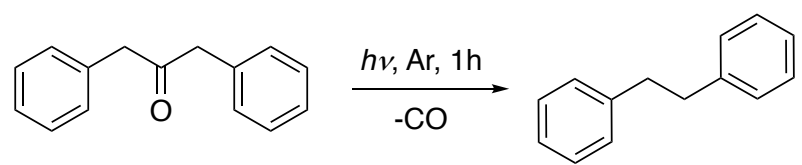

Scheme 3. Reaction of dibenzyl ketone upon exposure to UVB light forms free benzyl radicals that undergo head-to-head coupling exclusively.

Further, this arylation of benzylic radicals (i.e., formation of new $\mathrm{C}\left(\mathrm{sp}^{3}\right)-\mathrm{C}\left(\mathrm{sp}^{2}\right)$ bonds) can be photo-controlled to become essentially the only product-forming reaction (vide infra). For instance, controlling the irradiance it is possible to tune the reaction, and as the illumination decreases ring coupling can become essentially quantitative, a process that we demonstrated in detail for $p$-xylene, a simpler system where ring recombination yields a single product (Scheme 1).

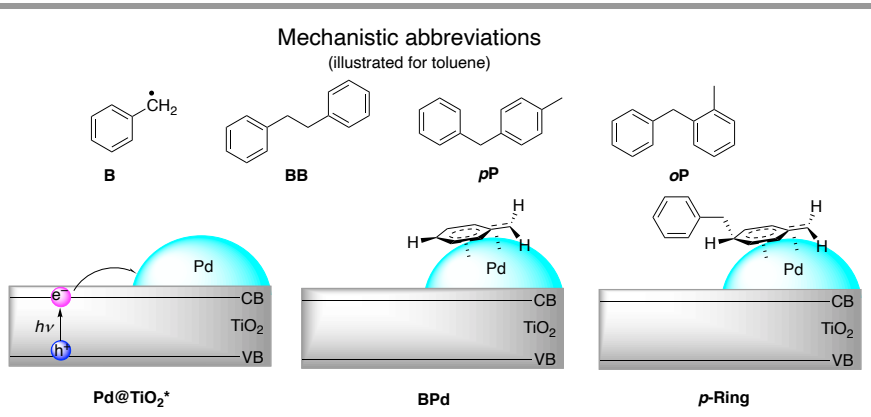

Chart 1. Abbreviations for the different species proposed in the reactions of toluene. Note for $\boldsymbol{p}$-Ring - the ring coupling product before aromatization and before release from the surface - that a similar species (not shown), o-Ring, exists in the case of ortho coupling. The same nomenclature is used for $p$-xylene, except that only one ring product $(o P)$ is formed.

Table 1. Conversion, moles of products and fraction of $\boldsymbol{O P}$ for the reaction of $p$-xylene using different catalyst. ${ }^{a}$

\begin{tabular}{ccccc}
\hline Catalyst & $\begin{array}{c}\text { Conversion } \\
(\mu \mathrm{mol})\end{array}$ & $\mathrm{n}_{\text {oP }}(\mu \mathrm{mol})$ & $\mathbf{n}_{\mathrm{BB}}(\mu \mathrm{mol})$ & oP (\%) \\
\hline $\mathrm{TiO}_{2}$ & $0.36 \pm 0.02$ & $\mathrm{ND}$ & $0.353 \pm 0.007$ & $\mathrm{ND}$ \\
$\mathrm{Au} @ \mathrm{TiO}_{2}$ & $0.5 \pm 0.1$ & $0.011 \pm 0.001$ & $0.5 \pm 0.1$ & $2 \pm 3$ \\
${\mathrm{Pd} @ \mathrm{TiO}_{2}}$ & $0.6 \pm 0.1$ & $0.108 \pm 0.002$ & $0.4 \pm 0.1$ & $21 \pm 5$ \\
\hline
\end{tabular}

a Reaction conditions: $4 \mathrm{~mL}$ of $p$-xylene, $20 \mathrm{mg}$ of catalyst under Ar atmosphere and UVA irradiation $\left(16 \mathrm{Wm}^{-2}\right.$ ) for $1 \mathrm{~h}$. Number of moles estimated by GC-MS using 3,5di-tert-butyltoluene as external standard. All values are duplicates (except the runs with $\mathrm{Pd} @ \mathrm{TiO}_{2}$ are triplicates). ${ }^{\mathrm{b}}$ products, including aldehyde formation. ND: not detected.

The selectivity towards $\mathrm{C}\left(\mathrm{sp}^{3}\right)-\mathrm{C}\left(\mathrm{sp}^{2}\right)$ coupling could be explained using PRE theory. The PRE was first described by Ingold and Fisher, and usually requires the simultaneous formation of a persistent and a transient radical. ${ }^{11}$ Here a persistent radical cannot recombine, or the process is severely hindered. In classical examples one of the radicals is persistent - e.g., TEMPO- while other transient radicals can react with either the persistent or other transient radicals, including selfrecombination. Living free radical polymerization is a typical example where this kinetic behaviour applies. ${ }^{14,}$ 15,16 While ring coupling products are unusual, in the specific case of DBK Ramamurthy et al. have detected ring coupling products between benzyl and phenyl acetyl radicals when confined within zeolite cavities. ${ }^{17}$ In our system, we proved the formation of free benzylic radicals by trapping experiments with TEMPO (Figure S8). These photogenerated benzylic radicals could bind to the PdNP, extending the radical lifetime - thus, forming a pseudo-persistent radical. The idea of PRE promoted by metal centres has been studied before in homogeneous phase, where the metal centre can prolong the radical lifetime. ${ }^{18-21}$ In our 
system we attribute persistent radical character to BPd (Chart 1) -we note that the substitution of $\mathrm{Au}$ for $\mathrm{Pd}$ also indicates PRE (Tables 1, S2-S3). In BPd, reactivity at the benzylic position is prevented by bonding at the metal surface and, thus, attack from benzylic radicals approaching from solution is directed to other positions in the aromatic ring. In a typical case where the persistent radical is not introduced at the beginning of the reaction, but rather formed as the reaction progresses (as is the case here), there is a brief period during which normal recombination (leading to $\mathbf{B B}$ ) dominates while the less reactive (and in this case less mobile) BPd accumulates, but soon becomes concentrated enough to dominate radical-radical reactions. A proposed mechanism is outlined below, where it is assumed that free $\mathrm{Pd} @ \mathrm{TiO}_{2}$ is not the limiting reagent and that no PdNP hosts more than one benzylic radical. As we note below, ideal conditions to favour ring coupling involve low light intensities where these conditions are readily met, Scheme 4.

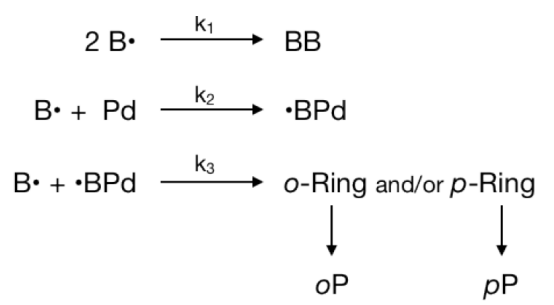

Scheme 4: Mechanism relating to the self-reactions of benzylic radicals $(B \bullet)$ in the presence of $\mathrm{Pd} @ \mathrm{TiO}_{2}(\mathrm{Pd})$.

Kinetics considerations lead us to believe the benzyl radical formation is not the rate-determining step of the reaction and, therefore, we can use the steady-state approximation to explain the selectivity of the reaction as dictated by two competitive pathways (i) radical recombination (BB) and (ii) formation of persistent radical species (BPd). Under steadystate conditions the following equations can describe the kinetics of the formation of ring-coupling products:

$$
\begin{aligned}
\mathrm{k}_{3}[\mathrm{~B} \cdot][\cdot \mathrm{BPd}] & =\mathrm{k}_{2}[\mathrm{~B} \cdot][\mathrm{Pd}] \\
\mathrm{k}_{3}[\cdot \mathrm{BPd}] & =\mathrm{k}_{2}[\mathrm{Pd}] \\
{[\cdot \mathrm{BPd}] } & =\mathrm{k}_{2}[\mathrm{Pd}]
\end{aligned}
$$

and the selectivity can be described as:

$$
\begin{aligned}
& \text { Selectivity }=S=\frac{\text { Head-to-Ring coupling }}{\text { Head-to-head coupling }} \\
& \qquad S=\frac{\mathrm{k}_{3}[\mathrm{~B} \cdot][\cdot \mathrm{BPd}]}{\mathrm{k}_{1}[\cdot \mathrm{B}]^{2}}=\frac{\mathrm{k}_{3}[\cdot \mathrm{BPd}]}{\mathrm{k}_{1}[\cdot \mathrm{B}]}=\frac{\mathrm{k}_{2}[\mathrm{Pd}]}{\mathrm{k}_{1}[\cdot \mathrm{B}]}
\end{aligned}
$$

Clearly, the concentration of Pd is crucial to favour ring coupling processes (Figure 1). Rather than the total concentration of Pd, or the concentration of PdNP, we believe that the number of surface $\mathrm{Pd}$ atoms $\left(\mathrm{N}_{\mathrm{s}}\right)$ could be the key parameter, something that is also in line with computational studies (vide infra). Several batches prepared - using the same protocol-yield slightly different materials (Table 2 ) and allow the simultaneous evaluation of irradiance and surface Pd population, calculated as described in the SI, Figure 1.
Table 2. Metal loading, particle size and superficial Pd atoms of three different Pd@ $\mathrm{TiO}_{2}$ batches.

\begin{tabular}{cccc}
\hline & & & \\
\hline Batch & Pd loading $^{\mathrm{a}}$ & $\mathrm{D}^{\mathrm{b}}$ & $\mathrm{Ns}^{\mathrm{c}}$ \\
\hline $\mathrm{A}$ & 2.3 & $2.1 \pm 0.9$ & 2.3 \\
$\mathrm{~B}$ & 2.7 & $1.3 \pm 0.5$ & 4.2 \\
$\mathrm{C}$ & 3.4 & $1.6 \pm 0.8$ & 4.4 \\
\hline Average & 2.6 & 1.7 & 3.7 \\
\hline
\end{tabular}

a Metal loading (wt \%) determined by ICP-OES. b D: PdNP diameter (nm) determined by TEM imaging. ${ }^{c}$ NS: surface Pd atoms $(\mu \mathrm{mol} / 20 \mathrm{mg})$, see calculation in SI.

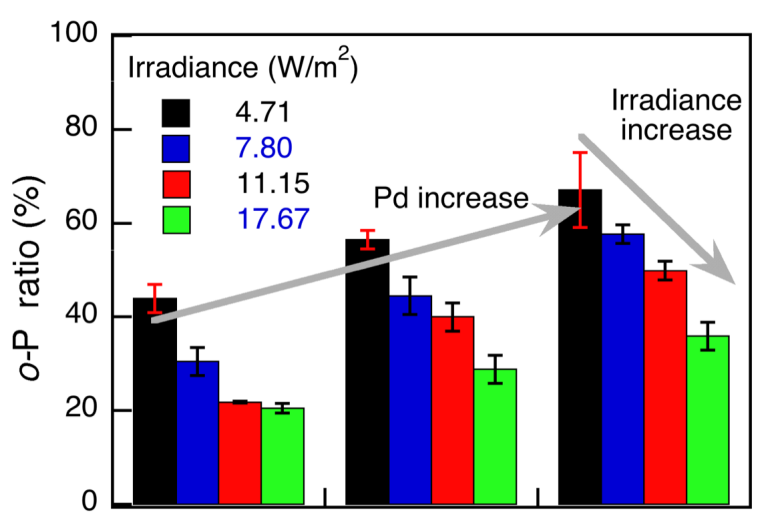

(A) 2.3

(B) 4.2

(C) 4.4 $\mathrm{N}_{\mathrm{S}}(\mu \mathrm{mol} / 20 \mathrm{mg})$

Figure 1. Effect of Pd concentration $\left(\mathrm{N}_{\mathrm{S}}\right)$ and of irradiance on the selectivity towards head-to-ring coupling product (o-P) for $\mathrm{p}$-xylene photocatalyzed by UVA for 3 different batches of $\mathrm{Pd} @ \mathrm{TiO}_{2}$ (table 1).

Additionally, as the selectivity is dictated by the formation of benzylic radicals, the irradiance used can drastically change the reaction product ratio (Figure 2). To establish this, Figure 3 shows the product $(\boldsymbol{O P})$ ratios obtained upon irradiation using different light intensities. Further, we show the raw data for different Pd@ $\mathrm{TiO}_{2}$ batches (Table S4) and a solid curve based on the simultaneous analysis of the three sets with the condition imposed that the fraction of head-to-ring coupling cannot exceed $100 \%$. The dashed line is the calculated fraction of headto-head product (BB). The trend in Figure 3 suggests that when the irradiance decreases, less benzylic radicals are free in solution, thus, leading to head-to-ring coupling competing favourably with head-to-head reaction, under conditions suitable for PRE. 


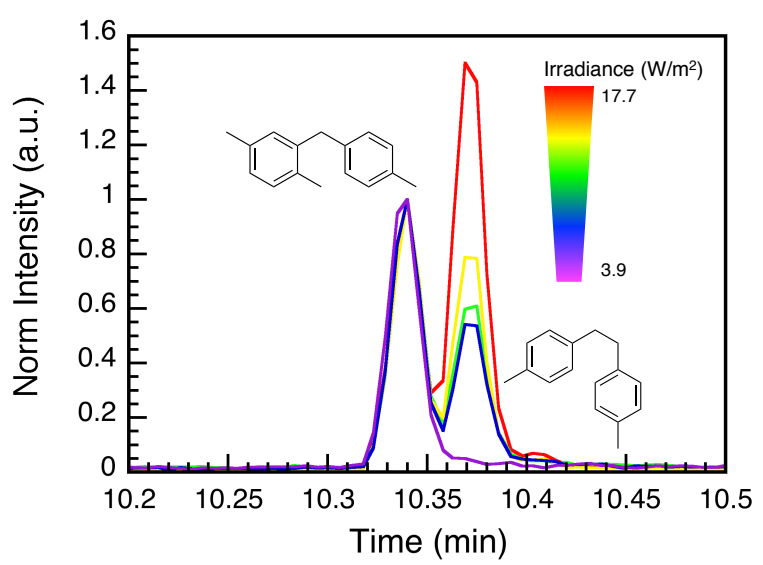

Figure 2. Chromatograms showing the peaks corresponding to the formation of head-toring and head-to-head coupling products for $p$-xylene using catalyst $C$.

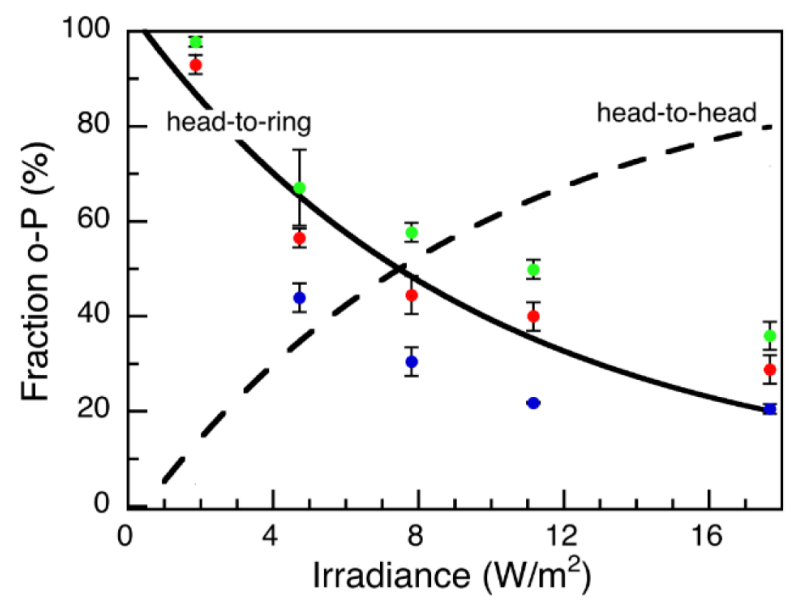

Figure 3. Effect of irradiance on the fraction of head-to-ring coupling $(o P)$ products for $p$

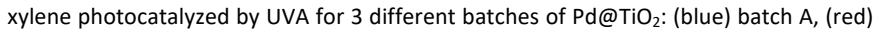
batch $B$, and (green) batch $C$. The average line shows a global fit for the 3 catalysts with the condition that the yield cannot exceed $100 \%$. See Table S3 for more details.

\section{Computational studies}

To gain further insights into the mechanism, we performed DFT calculations to study the interaction between benzylic radicals and a cluster of palladium atoms. First, we characterized free benzyl radicals using PBE level of theory. The benzyl radical shows $C_{2 v}$ symmetry with the spin unpaired localized mainly in the carbon of the alkyl group (+0.59) and partially in the ortho $(+0.19)$ and para $(+0.19)$ positions of the aromatic ring (+0.19). The partial charge of carbon atoms in the aromatic ring is of similar magnitude $(\approx-0.12)$, while the carbon of the alkyl group shows the highest negative charge $(-0.31)$ due to the covalent bond with two hydrogen atoms (see Figure 4). Similar results have been found at BP86-D3/Def2-TZVP level of theory (see Figure 5). For comparison, we also explore the symmetry and charge density distribution in benzyl anion and benzyl cation. The partial charge of carbon atoms in the benzyl anion aromatic ring are centred in the para and ortho positions (-0.18 and -0.15 respectively) and as expected, the highest negative charge is located at the alkyl carbon. On the other hand, the benzyl cation localises the charge at the ortho positions $(-0.13)$, whereas the alkyl carbon is also slightly charged (-0.09).

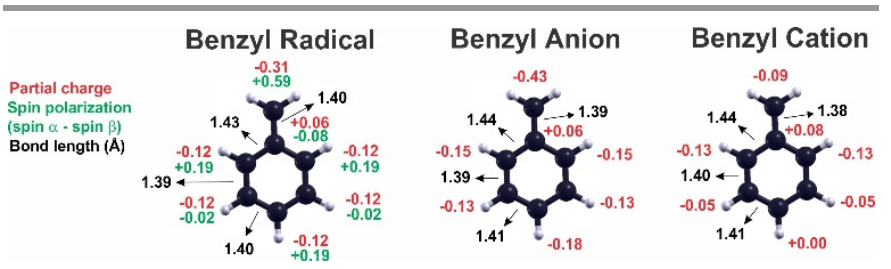

Figure 4. Optimized structure of benzyl radical, anion and cation computed at the PBE level of theory.
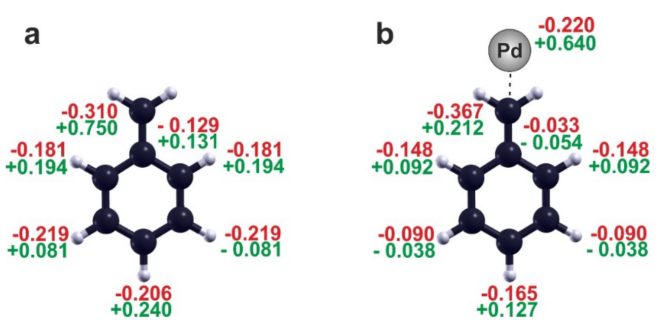

Figure 5. Optimized structure of benzyl radical and the corresponding complex with one atom of palladium computed at the UBP86-D3/Def2-TZVP level of theory showing charge density (in red color) and spin polarization (in green color) on carbon atoms.

Then, we analysed the interaction of benzyl structures with the metal surface. As both $\mathrm{Pd}$ and $\mathrm{Au}$ metal nanoparticles have shown some selectivity towards the head-to-ring coupling, we have performed computational studies using both elements. A cluster of $\mathbf{2 2 5}$ atoms of Pd (or $\mathrm{Au}$ ) was taken as theoretical model for studying the interaction between the benzyl radical and the metal nanoparticles since the corresponding size $(\sim 1.9$ $\mathrm{nm})$ nicely resembles the experimental dimension of the NPs prepared $(\sim 2.0 \mathrm{~nm})$. The average Pd-Pd ( 2.74 $\AA$ ) —and Au-Au $(\sim 2.84 \AA)-$ bond lengths in $\mathrm{Pd}_{225}$ and $\mathrm{Au}_{225}$ agree well with literature values (Figure S9). ${ }^{22,} 23$ Different starting geometries were considered when studying the interaction between benzyl radical with metal clusters, including radical interacting at the center of [111] facet of NP and/or at the edge of the metal cluster. Five minimum structures have been found for the complexes between benzyl radical and $\mathrm{Pd}_{225}$ cluster, while four structures have been computed in case of $\mathrm{Au}_{225}$ cluster (Figure S10-S11). The highest adsorption energy was computed when the benzyl radical approaches the edge of the NP for both Pd ($\left.30.3 \mathrm{kcal} \mathrm{mol}^{-1}\right)$ and Au clusters $\left(-26.9 \mathrm{kcal} \mathrm{mol}^{-1}\right)$. However, it is worth noting that the average adsorption energy is higher when the benzyl radical interacts with $\mathrm{Pd}_{225}\left(-27.7 \mathrm{kcal} \mathrm{mol}^{-1}\right)$ than with $\mathrm{Au}_{225}\left(-21.2 \mathrm{kcal} \mathrm{mol}^{-1}\right)$. This is probably due to the different packed plane [111] between $\mathrm{Pd}_{225}$ and $\mathrm{Au}_{225}$ clusters. In the benzyl radical, the length between the carbon of the alkyl group and the carbons at the ortho ( $2.47 \AA$ ) and para positions (4.24 $\AA$ ) resembles better the atom organization found in $\mathrm{Pd}_{225}$ than that of $\mathrm{Au}_{225}$ (Figure S9). Indeed, in case of $\mathrm{Pd}_{225}$, the benzyl radical is interacting not only at the alkyl group but also with aromatic ring, and as result the radical "follows" the [111] surface of $\mathrm{Pd}$. In the case of $\mathrm{Au}_{225}$, the main interaction is only between the alkyl group of benzyl radical with gold atom, while no significant interaction is found with the aromatic ring (the 
benzyl radical is tilted respect to the [111] surface of $\mathrm{Au}_{225}$ ). Overall, the average adsorption energy between benzyl radical and $\mathrm{Pd}_{225}\left(-27.7 \mathrm{kcal} \mathrm{mol}^{-1}\right)$ - where the most stable structure is shown in Figure 6 - agrees with the values of dissociation bonding energies reported for alkoxyamines (e.g., benzylTEMPO) of relevance in "living" free radical polymerization. ${ }^{24}$ Thus, the binding energy is such that benzyl radicals can be in equilibrium with their persistent form (i.e., BPd), with the latter being thermally stable at temperatures below $373 \mathrm{~K}$.
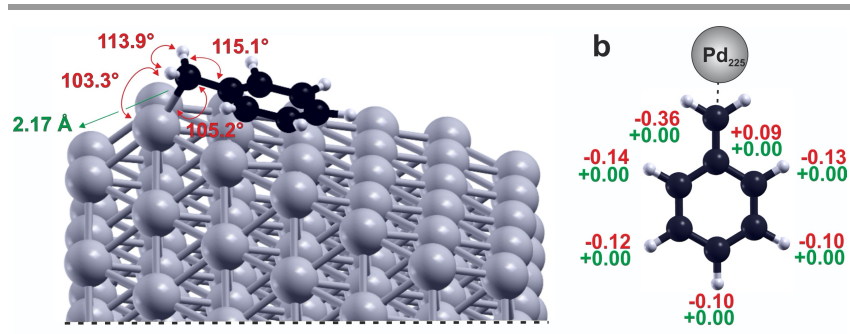

$E_{\text {ads }}=-30.3 \mathrm{kcal} \mathrm{mol}^{-1}$

Figure 6. a) Calculated structure of benzyl radical on $\mathrm{Pd}_{225}$. b) Löwdin population analysis showing the charge density (in red color) and spin polarization (in green color) on carbon atoms.

Importantly, by analysing the spin polarization, the interaction between the benzyl radical either with $\mathrm{Pd}_{225}$ and $\mathrm{Au}_{225}$ results that the unpaired spin is not anymore concentrated in the organic moiety, but rather delocalized along the metal atoms of NP. An increase of electron density compared to the "free" benzyl radical is computed at the ortho and para positions especially in case of $\mathrm{Pd}_{225}$, while in meta positions a decrease of negative charge is observed. Thus, it can be inferred that the $\mathrm{C}-$ $C$ coupling products observed experimentally may arise from the electrophilic attack of a "free" benzyl radical to the ortho or para positions of a benzyl species adsorbed on the metal nanoparticle surface. In summary, it seems that the metal nanoparticle (especially $\mathrm{Pd}$ ) "activate" the ortho and para positions of benzyl species towards an electrophilic attack.

Regarding the detailed geometry of the species formed, computational studies showed significant pyramidalization of the benzylic carbon upon binding to Pd. The length of the C-Pd bond (when considering a Pd atom at the edge of the cluster, see Figure 6) is $2.17 \AA$. Further, the bond contains $c a$. $20 \% \mathrm{~s}$ character. In addition, the benzyl $\mathrm{C}-\mathrm{C}$ bond is stretched from $1.402 \AA$ in the free benzyl radical to $1.439 \AA$ in the BPd species, whilst the $\mathrm{C}$ in $\mathrm{CH}_{2}$ is slightly pyramidal, Figure 6 and Table 3. This result is also confirmed by DFT calculations (BP86-D3/Def2TZVP) with a single atom of $\mathrm{Pd}$, in which the benzyl radical loses spin density at those positions while the palladium atom acquires it (Figure 6). As in case with $\mathrm{Pd}_{225}$, a C-Pd bond is formed between the $\mathrm{C}$ of $\mathrm{CH}_{2}$ (which adopts a slight pyramidal geometry) and $\mathrm{Pd}$ atom releasing $47.4 \mathrm{kcal} \mathrm{mol}^{-1}$. The newly orbital of carbon in the C-Pd now incorporates $9.9 \%$ of $\mathrm{S}$ character, similarly to the results computed with $\mathrm{Pd}_{225}$ cluster. In contrast to the $\mathrm{Pd}_{225}$ calculations, all the carbon in the aromatic rings lose charge density on interaction with single $\mathrm{Pd}$ atom, which may indicate the importance of Pd nanoparticle on activating the ortho and para positions towards an electrophilic attach from benzyl radical. Instead, the spin density decreases remarkably upon the interaction with $\mathrm{Pd}$ atom, especially in ortho and in para position as well as in the $\mathrm{C}$ of $\mathrm{CH}_{2}$, while the $\mathrm{Pd}$ atom acquires it.

Table 3. Table showing the computed $\mathrm{C}-\mathrm{C}$ bond length $\left(\mathrm{C}\left(\mathrm{sp}_{2}\right)-\mathrm{C}\right.$ (methylene)) for toluene, benzyl radical and the corresponding complexes with metal clusters ( $\mathrm{Pd}_{225}$ and $\left.\mathrm{Au}_{225}\right)$.

\begin{tabular}{cc}
\hline Species & $\mathrm{C}-\mathrm{C}$ bond length $(\AA)$ \\
\hline Toluene & 1.509 \\
Free benzyl radical & 1.402 \\
Benzyl radical-Pd 225 & $1.439^{*}$ \\
Benzyl radical-Au ${ }_{225}$ & $1.453^{*}$ \\
\hline${ }^{*}$ Average C-C bond value of the structures shown in Figure S10 and S11.
\end{tabular}

Overall, the benzylic species found on the Pd nanoparticle surface show no spin polarization, a charge density distribution and pyramidalization that can resemble the benzyl anion molecular structure. These results are consistent with metalpromoted persistency for benzylic radicals. Therefore, the preference for reaction at the ring when benzylic radicals attack the BPd complex may be assisted by the slight increase in electron density at the ortho and para positions, along with the fact that $\mathrm{C}$ at $\mathrm{CH}_{2}$ is already tetravalent and slightly pyramidal, and thus, an unlikely site for additional bonding. We note (see Figures S10 and S11) that the ring always stabilizes parallel to the Pd surface, consistent with $\boldsymbol{\pi}$ interactions with the surface. The $\mathrm{TiO}_{2}$ support clearly plays a role in the formation of benzylic radicals, but it is closer to an spectator in the reactions that lead to head-to-ring product formation.

\section{Conclusions}

We have shown that PdNP can change completely the regioselectivity of benzylic radical reactions, favouring nontraditional radical couplings of potential interest in organic synthesis. The Ingold-Fischer PRE theories provide a reliable paradigm for the interpretation of the preference for head-toring coupling. The concepts developed here for simple methylbenzenes may enable regiochemical control of radicalradical reactions in many other systems.

\section{Conflicts of interest}

There are no conflicts to declare.

\section{Acknowledgment}

This work was supported by the Natural Sciences and Engineering Research Council of Canada, the Canada Foundation for Innovation, the Canada Research Chairs Program, Canada's International Development Research Centre (IDRC) and the German National Academy of Sciences Leopoldina (Grant No. LPDS 2017-15 to P.C.).

\section{Corresponding Authors}

AEL: anabel.lanterna@icloud.com 
JCS: jscaiano@uottawa.ca

\section{References}

1. C. Walling, Free radicals in solution, Wiley, California, USA, 1957.

2. B. M. Trost, Science, 1991, 254, 1471.

3. H. Yi, G. Zhang, H. Wang, Z. Huang, J. Wang, A. K. Singh and A.

Lei, Chem. Rev., 2017, 117, 9016-9085.

4. M. Ueda, E. Kondoh, Y. Ito, H. Shono, M. Kakiuchi, Y. Ichii, T. Kimura, T. Miyoshi, T. Naito and O. Miyata, Org. Biomol. Chem., 2011, 9, 2062-2064.

5. R. Vanjari and K. N. Singh, Chem. Soc. Rev., 2015, 44, 8062-8096.

6. M. B. Zhou, C. Y. Wang, R. J. Song, Y. Liu, W. T. Wei and J. H. Li, Chem. Commun., 2013, 49, 10817-10819.

7. A. Hainer, N. Marina, S. Rincon, P. Costa, A. E. Lanterna and J. C. Scaiano, J. Am. Chem. Soc., 2019, 141, 4531-4535.

8. E. Wada, T. Takeuchi, Y. Fujimura, A. Tyagi, T. Kato and H. Yoshida, Catal. Sci. Technol., 2017, 7, 2457-2466.

9. K. J. Romero, M. S. Galliher, D. A. Pratt and C. R. J. Stephenson, Chem. Soc. Rev., 2018, 47, 7851-7866.

10. L. L. Zhang, H. Yi, J. Wang and A. W. Lei, Green Chem., 2016, 18, 5122-5126.

11. A. Bravo, H. R. Bjorsvik, F. Fontana, L. Liguori and F. Minisci, J. Org. Chem., 1997, 62, 3849-3857.

12. P. B. Merkel, P. Luo, J. P. Dinnocenzo and S. Farid, J. Org. Chem., 2009, 74, 5163-5173.

13. A. Elhage, A. E. Lanterna and J. C. Scaiano, ACS Sust. Chem. Eng., 2018, 6, 1717-1722.

14. H. Fischer, Chem. Rev., 2001, 101, 3581-3610.

15. C. J. Hawker, J. Am. Chem. Soc., 1994, 116, 11185-11186.

16. D. H. Solomon, E. Rizzardo, and P. Cacioli, U. S. Patent 4581429

(Apr. 8, 1986); Chem. Abstr., 102, 221335q (1985)

17. V. Ramamurthy, D. R. Corbin, N. J. Turro, Z. Zhang and M. A.

Garcia-Garibay, J. Org. Chem., 1991, 56, 255-261.

18. J. C. Scaiano, W. J. Leigh and G. Ferraudi, Can. J. Chem., 1984,

62, 2355-2358.

19. H. Cohen and D. Meyerstein, Inorg. Chem., 1974, 13, 2434

2443.

20. M. Freiberg and D. Meyerstein, J. Chem. Soc., Faraday Trans., 1977, 73, 622-631.

21. M. Freiberg and D. Meyerstein, J. Chem. Soc., Faraday Trans., 1980, 76, 1825-1837.

22. K. P. McKenna and A. L. Shluger, J. Phys. Chem. C, 2007, 111, 18848-18852.

23. I. V. Yudanov, A. Genest and N. Rösch, J. Cluster Sci., 2011, 22, 433-448.

24. W. G. Skene, S. T. Belt, T. J. Connolly, P. Hahn and J. C. Scaiano, Macromolecules, 1998, 31, 9103-9105. 